\title{
Problemas de gestión social y rendimiento económico en pymes que forman emporios tipo gamarra en el Perú periodo 2006-2015
}

\author{
Social management problems and economic performance in \\ peruvian smes belonging to gamarra-type trading centers \\ (2006-2015)
}

\author{
Nancy Esquives ${ }^{1}$ \\ 'Escuela de Post Grado, Facultad de Ciencias Económicas \\ Universidad Nacional Mayor de San Marcos, Lima Perú
}

Recibido : 27/04/2017 Aceptado: 08/07/2017

\begin{abstract}
RESUMEN
Hoy en día se requiere conseguir, además de la viabilidad económica y ambiental, la viabilidad social en toda actividad empresarial. Los numerosos conflictos en torno a diferentes actividades económicas, en diferentes zonas del país, demuestran claramente la álgida importancia de conseguir la aceptación de la población, incluyendo a las autoridades locales del área en la cual se realiza dicha actividad.

Las Pymes del Emporio Gamarra no están ajenos a esta realidad, por lo tanto estas empresas necesitan conseguir y mantener la viabilidad social en el entorno donde desarrollan su actividad.

Si bien es cierto esta aceptación no constituye una garantía del éxito empresarial, sí contribuye a crear las condiciones para el establecimiento de relaciones de buena vecindad que facilitan la gestión social y ambiental.

Dadas las condiciones socioeconómicas que caracteriza a este sector, para lograr dicha viabilidad social se requiere que los líderes de los titulares de las Pymes tengan en cuenta desde el inicio de la actividad que la viabilidad social requiere de un compromiso sostenido en el tiempo. Este debe reflejarse en los esfuerzos compartidos en la planificación de acciones de relacionamiento y apoyo con la población local y sus autoridades.

La aceptación de la actividad de las Pymes del Emporio Gamarra por parte de la población local no es un proceso natural, por lo se requiere desarrollar esfuerzos específicos, bien pensados, planificados y que cuenten con los recursos (financieros, técnicos y personal idóneo) suficientes para lograrlo. Idealmente estos esfuerzos deberían permitir desarrollar alianzas estratégicas entre la comunidad, las autoridades locales y las empresas que se concreten en la identificación, definición e implementación de proyectos de desarrollo sostenible local.

EI presente artículo denominado "PROBLEMAS DE GESTION SOCIAL Y RENDIMIENTO ECONOMICO EN PYMES QUE FORMAN EMPORIOS TIPO GAMARRA EN EL PERU”, es una importante herramienta que contiene conceptos básicos acerca de lo que es la gestión social, la gestión de conflictos, la responsabilidad social, el desarrollo sostenible y la comunicación, entre otros, con el fin de establecer un nivel de conocimiento estandarizado que contribuya a la formulación de estrategias de gestión social que permitan compatibilizar el desarrollo local, el bienestar de la población y el éxito empresarial en la zona de influencia de las Pymes del Emporio Gamarra.
\end{abstract}

Palabras Claves: Gestión Social

\section{ABSTRACT}

In the present context, all business activities need to reach, in addition to economic and environmental viability, social legitimacy. The numerous conflicts sparked by economic activities in different areas of the country clearly demonstrate the critical importance of getting the acceptance of the populations and the local authorities of the area in which the activity is performed.

The SMEs of the Gamarra trading center are not oblivious to this reality, and realize that they need to obtain and maintain their social viability in the environment where they operate.

While acceptance is not a guarantee of business success, it contributes to create the conditions for the good neighborly relations that facilitate social and environmental management.

* Correspondencia:

E-mail: nchunga@hotmail.com 
Given the socioeconomic conditions that characterize this sector, in order to achieve this social viability, SME leaders must keep in mind from the start that social viability requires a sustained commitment over time. This must be reflected in shared efforts during the planning of the relationship and the support activities with the local populations and their authorities. Support by the local population for SME activities in the Gamarra trading center is not a natural process. To achieve it, it is necessary to develop specific, well planned activities and to count with sufficient financial, technical and human resources. Hopefully, these efforts should allow the building of strategic partnerships between the community, the local authorities and the businesses, which would bring forward the identification, definition and implementation of local sustainable development projects.

The present article, called "Social management problems and economic performance in Peruvian SMEs belonging to Gamarratype trading centers (2006-2015)", is an important tool containing basic concepts about what are social management, conflict management, social responsibility, sustainable development and communication, among others. Its goal is to establish a standardized level of knowledge to contribute to the development of social management strategies, in order to reconcile local development, the population welfare and business success, within the area of influence of the SMEs of the Gamarra trading center.

Keywords: Social Management

\section{INTRODUCCIÓN}

Son muchos países los que les gustaría poseer un emporio comercial como es Gamarra, por lo que muchos de ellos intentan copiarlo como modelo de exportación, tal es el caso de la llegada de una delegación de 22 empresarios venezolanos, para analizar oportunidades de inversión en nuestro país.

Estos empresarios, estaban particularmente interesados en construir en la ciudad de Maracaibo un complejo similar al de Gamarra.

Esta noticia de que el emporio comercial textil más grande del Perú, sea considerado un modelo de exportación nos llena de orgullo. Sin embargo, aún existen problemas pendientes que Gamarra, con sus 17.000 microempresas y pequeñas empresas y ventas anuales por US\$800 millones, deberá superar si aspira a afrontar con éxito los retos que se le presentan.

El conglomerado textil tiene un perfil netamente de microempresa: un $95,7 \%$ de las empresas tiene de uno a diez trabajadores ( $y$ de ese porcentaje el $82 \%$ opera con uno a cuatro trabajadores).

Otro dato que llama la atención es que el $63,5 \%$ de las mypes tiene hasta cinco años de actividad, es decir han sido creadas entre el 2003 y el 2007. Y aunque es cierto que la cifra podría indicar que la mortalidad empresarial todavía es alta, porque la mayoría no cuenta con información ni con una plataforma de acceso al mercado (en el Perú por cada 100 microempresas que se crean 70 desaparecen al año).

El conglomerado invierte y labora con gente básicamente joven: el 52\% de los empresarios tiene entre 18 y 34 años de edad. Mientras que el $26 \%$ de empresarios tiene entre 35 y 44 años. "Las cifras confirman que una parte importante del grupo que conduce Gamarra son hijos e hijas de los migrantes de diferentes departamentos del Perú, que llegaron a Lima para formar el emporio.

Los principales problemas de gestión que han identificado en el Emporio Comercial Gamarra son: las ventas bajas, el no saber cómo enfrentar a la competencia y escasa información de mercado y muy importante es que no existe una adecuada gestión social en las Mypes, por lo que ocurren constantes fricciones entre las empresas y su zona de influencia.

Es claro que hay una ausencia significativa de una adecuada gestión capacitación, por lo que no existe aún en el emporio una comunidad con espíritu emprendedor para generar un cambio social, para responder a la búsqueda de la superación de la pobreza, e igualmente para que se adquieran destrezas para abordar un entorno de turbulencia cuyos componentes políticos, económicos, socio-culturales, ambientales y tecnológicos no favorecen los procesos de desarrollo sociales; de ahí, que se requiere afianzar los lazos comunitarios, recuperar los valores colectivos y recuperar la identidad cultural, así mismo, implica la convergencia de intereses y necesidades comunes, finalidades humanas concertadas.

\subsection{PROBLEMÁTICA}

¿De qué manera los problemas de gestión social afectan el rendimiento económico en Pymes que forman Emporios tipo Gamarra en el Perú?

\subsection{JUSTIFICACIÓN}

Una adecuada gestión social permite desarrollar una capacidad empresarial orientada a responder a la complejidad que atraviesan los niveles de interrelación e interacción de los actores, sectores, poderes y voluntades que realcen la importancia de las dinámicas que viven las comunidades quienes son las que generan el desarrollo de todo tipo.

En mi criterio, la investigación se justifica desde el punto de vista teórico porque el conocimiento científico del problema permitirá, establecer cuáles son los problemas de gestión social que afectan el rendimiento económico en pymes que forman emporios tipo Gamarra en el Perú sobre la base de ese conocimiento, proceder a elaborar, aprobar y poner en 
vigencia propuestas a partir de políticas y estrategias en forma científica, técnica, eficiente y eficaz.

La gestión social en las Pymes del emporio Gamarra permitirá a todos los actores sociales públicos y privados gestionar sus propias transformaciones particulares en colectivas, un contexto de civilidad moderna y desde un Estado Social de Derecho en donde las personas se sienten partícipes, y protagonistas de las relaciones sociales, no solo receptores de derechos, sino también garantías de obligaciones y nuevas responsabilidades sociales.

La gestión social posibilita el desarrollo de ciudadanía, la creación de una cultura de corresponsabilidad, de aceptación de la diferencia, del diálogo, de la información, de la formación, y la aplicación del conocimiento para el progreso, para el desarrollo, para la inclusión social y para la transformación, soportada en herramientas como la planeación estratégica, el desarrollo humano, la organización, la participación, el trabajo en equipo, el liderazgo y la investigación que permitan dotar a las comunidades de los elementos teóricos y prácticos y así lograr la implementación de políticas públicas para materializar el cambio social y reducir la pobreza.

\subsection{OBJETIVOS}

Determinar de qué forma los problemas de gestión social afectan el rendimiento económico en pymes que forman emporios Gamarra en el Perú.

Establecer cuáles son los elementos de la gestión social que influyen en el rendimiento económico en Pymes que forman emporios tipo Gamarra en el Perú.

Determinar de qué manera se puede mejorar la gestión social de las Pymes que forman emporios tipo Gamarra en el Perú para influir en su rendimiento económico.

Definir los stakeholders que las Pymes que forman emporios tipo Gamarra en Perú deben considerar para desarrollar su gestión social.

\section{MARCO TEORICO}

\subsection{Emporio de Gamarra}

En el Emporio Comercial de Gamarra, ubicado en el distrito de La Victoria, existen más de 24 mil establecimientos; de los cuales 15 mil 526 establecimientos están dedicados a la actividad de comercio, cifra que representa el $64,0 \%$ del total de unidades económicas; informó el Instituto Nacional de Estadística e Informática (INEI mayo del 2015). Dicha zona empresarial que el presente año cumple cincuenta años alberga a la clase emprendedora de nuestro país.

En Gamarra también se registraron establecimientos manufactureros en número de $5 \mathrm{mil}$ $632(23,2 \%)$. El resto de establecimientos se dedican a las actividades de transportes y almacenamiento (7,4\%), alojamiento y servicios de comida $(2,3 \%)$ y otras actividades de servicios (3,1\%).

Son características del Emporio Gamarra:

- Mayoría de establecimientos son microempresas

- En Gamarra trabajan más de 51 mil personas

- Ocho de cada diez personas laboran en textiles y prendas de vestir

- Tres quintas partes de trabajadores son mujeres

- Más del $80 \%$ de trabajadores tiene entre 18 y 40 años de edad

- Uno de cada cinco trabajadores tiene estudios superiores

\subsection{Polvos Azules, la informalidad}

En el Perú, cuatro de cada 10 negocios son informales, los negocios informales se caracterizan principalmente por estar orientados al gran público masivo de escasos recursos económicos, (Estratos sociales $D$ y $E$ de la población), esto se observa principalmente en el sector del comercio y producción a menor escala; como el calzado, confecciones, alimentación, metal mecánica, etc. La informalidad compite casi en todos los sectores industriales y de servicio.

La particularidad del mercado informal, es que donde existe un emporio comercial, existe toda una gama de productos y servicios nuevos para todos los gustos y colores, existen grandes emporios comerciales de micro comerciantes informales como polvos azules en Lima, Avelino Cáceres en Arequipa y en otras ciudades importantes del país, los productos que venden los informales aparecen muchas veces con la misma velocidad con que desaparecen, otras constituyen alternativas baratas para los consumidores que son producidas en su mayoría por los informales.

En la actualidad el peso de la economía informal sigue en aumento, conforme sus productos y servicios empiezan a competir directamente con los de los empresarios formales, se vuelven una amenaza, cuando no se actúa de inmediato terminan reemplazando a los formales.

Ejemplos tenemos en los supermercados, por lo menos dos de ellos desaparecieron entre otros razones por el cinturón de informales que los asfixiaron, los restaurantes sufren esta amenaza constantemente muchos restaurantes, terminaron siendo reemplazados por la carretilla de comida o el carro sandwuchero, similares casos se observan en el sector de medianos productos plásticos, ladrilleras, etc.

\subsection{Perfil competitivo del informal}

El punto clave para entender la lógica competitiva de estos negocios, es saber; ¿Qué habilidades especiales demuestran estas personas para conectarse con el público consumidor que están más al tanto de sus necesidades?, ¿Porqué siempre están con el 
producto en el lugar adecuado y en el momento oportuno?, ¿Es el precio, realmente, la variable más importante?, ¿Los comerciantes no tienen más remedio que dejar a los informales ese gran segmento de la población?

En este artículo no se discute a los informales si es un tema social ó económico, sino se le mira como competidor directo para muchos negocios formales.

\subsection{Competidor cuenta con ventajas ya conocidas}

No tributan, no están sometidas a inspecciones de las autoridades o de instituciones del Estado. Cuentan con experiencia en algún sector industrial, o de servicios, en el cual ya trabajaron, involucra además a parte de su familia en el negocio.

Cuentan con un capital de trabajo limitado, los créditos que otorga lo hacen por la confianza, no cuentan como documentación legalmente válida para poder reclamar alguna deuda

Su principal motivación está basado en su deseo de mantenerse él y su familia, para esto debe vender, de esta manera cada instante de su trabajo, lo orienta a buscar aquello que puede vender fácilmente así él y su familia están involucrados en un constante monitoreo de oportunidades de ventas.

La mayoría están afiliados a asociaciones que constituyen verdaderos cercos que impiden establecerse como negocios formales porque consideran que así les va bien.

\subsection{Análisis competitivo}

Las empresas formales necesitan incorporar en su estrategia de marketing a los "informales" como competidores, para ello es importante analizar la lógica competitiva que usan los informales para diseñar, producir y comercializar sus productos y/o servicios, en base a este análisis se puede notar características comunes en ellos:

- Venden productos con imitaciones de otros que ya existen en el mercado

- Usan nombres similares a los de los modelos originales

- La calidad-precio es inferior a los de los originales

- Se expanden en lugares poco aparentes para comercializar sus productos o servicios

Polvos azules, el mercado de Lima donde todo es posible.

Una de sus fortalezas es sin duda, el buen manejo de la información de sus clientes, la lógica no les permite invertir en estudios de mercado, lo que hacen es explotar al máximo algunas variaciones o novedades en los gustos de los consumidores, a menudo descubiertos ya por otras empresas o personas, estas variaciones, los convierten en "modas" para luego producir grandes cantidades que terminan saturando el mercado.

\subsection{Posicionamiento}

Existe un estereotipo del informal Peruano, lo cual contribuye a su posicionamiento, esto al margen del concepto particular que tenga el consumidor de algún informal, así las palabras claves que están metidas en el inconsciente de los consumidores al referirse a un informal son; menor precio, y calidad aceptable.

Si bien la calidad de productos que ofrecen los informales, en la mayoría de los casos no resista el mínimo control de la calidad, sin embargo no olvidemos que el concepto mismo de la calidad está en la mente del consumidor, aquí no entra a tallar el significado objetivo de la calidad, sino la percepción de los consumidores, en es un elemento subjetivo, en este caso, la calidad, para este segmento de mercado, puede ser aceptable, el consumidor es consciente de que a determinados niveles de precios, no puede pedir más y se siente satisfecho con una transacción que su entender le parece justa o razonable.

Frente a este posicionamiento, la mayoría de empresas formales tratan de evitar a este gran segmento de mercado, e intentar migrar a otros segmentos que representen ingresos similares a los anteriores; sin embargo esta es una ventaja que puede ser aprovechada por la empresa formal.

\subsection{Estrategia competitiva}

Considerando que en determinados sectores industriales y de servicios no existen algunos con nombre propio, a quien a poder atribuir las cualidades positivas de los informales, se debe procurar ser el primero en penetrar en la mente del consumidor con un nombre al cual se le relacione con precios "baratos" y calidad aceptable.

Para esto no siempre funcionan las costosas campañas publicitarias, hay que tener en cuenta que el "gancho" que ofrecen los informales para atraer al público consumidor masivo es menor precio, variedad y agilidad, se debe transmitir este concepto en el medio de comunicación más usada por los consumidores de este segmento, que habitualmente es la radio, los paneles publicitarios.

El concepto del producto debe ser muy similar al que usa el informal y su mercado, la idea es que el consumidor no observe ninguna diferencia en variedad, rapidez, lo que su mercado llega a pedir, que sepa explotar las debilidades del informal, para poder diseñar estrategias integrales de acción en sus mercados.

Paralelamente desarrollar acciones efectivas de defensa contra la competencia desleal y piratería, aunque este tema se ha visto mucho en los últimos tiempos, incluso con apoyo de entidades del Estado, su 
efectividad no ha sido buena, en razón a que los informales tienen aun público consumidor masivo que sabe que un informal tiene menor precio y en dichos mercados puede encontrar de todo, En este punto se necesita ser creativo para vencer a tal cantidad de gente de que siempre ganara comprando al informal, el secreto estará en que el informal siempre y cuando esto sea cierto.

\section{ANTECEDENTES}

\subsection{Historia de Emporio}

Un emporio era un lugar que los comerciantes de una nación se habían reservado para sus intereses comerciales en el territorio de otra nación. Entre los emporios famosos se encuentran Sais, donde Solón llegó para adquirir el conocimiento de los egipcios; Elim, donde Hatshepsut mantenía su flota del Mar Rojo; Elat, actual Eilat, en donde Tebas se suministraba de materiales funerarios como tejido de lino, betún, nafta, incienso, mirra y amuletos de piedra tallada de Palestina, Canaán, Aram, Líbano, Hazor, Moab, Edom y la Península Arábiga desde Petra hasta Madian y Punt.

Los emporios funcionaban de forma muy similar a las colonias europeas de comercio en China.

En la Antigua Grecia se refiere tanto a las distintas ciudades-estado griegas y fenicias y puestos comerciales en Egipto, el norte de África, España, Gran Bretaña y la Península Arábiga. Están incluidas en este término ciudades como Avaris y Asuán en el Bajo Egipto y Tebas en el Alto Egipto, Elim y Eilat. Para los hititas se incluía Kanesh y Qadesh en la frontera del Líbano, Siria y Canaán y para los fenicios se incluían Gades, Cartago, Leptis Magna y Cirene, entre otros (aunque Cirene fue fundada por los griegos).

\subsection{Definición de Emporio Comercial}

Mercado en que se ofrecen bienes de diversos orígenes, por extensión, lugar o asentamiento importante por su actividad comercial. También se puede definir como firma o empresa de gran magnitud. Otra definición sería que son establecimientos comerciales de gran superficie, que vende al por menor artículos de diversos rubros, en especial indumentaria y menaje doméstico.

\subsection{Principales Emporios Comerciales en el Perú}

\section{a. Gamarra}

El Emporio Comercial de Gamarra, también conocido como "Gamarra", es un lugar de gran movimiento comercial principalmente relacionado a la industria de la moda y el textil, ubicado en el céntrico distrito de La Victoria en Lima, Perú.
Su crecimiento y desarrollo desde 1970 contiene historias de esfuerzo, persistencia y éxito. Gamarra ha servido de motivación y ejemplo para el desarrollo de la mediana y micro empresa peruana, ha permitido el surgimiento de grandes marcas y tiendas locales, y juega un rol trascendental en el desarrollo del sistema del Diseño de Moda y Textil en el Perú.

Hoy Gamarra concentra a más de 10 mil empresarios, 17 mil tiendas distribuidas en más de 144 galerías ubicadas en 20 manzanas del distrito. Además, ofrece un número cuantioso de servicios, insumos y otros productos relacionados a la industria textil, de confecciones y de moda a nivel nacional, regional e internacional.

A pesar de generar empleo, de contar con uno de los metros cuadrados de alquiler más altos del país y de contener historias de migración, emprendimiento y éxito, Gamarra también es asociado frecuentemente con la idea de una potencia comercial desorganizada donde se concentran el transporte desordenado, el incumplimiento de leyes de empleo, un aparente descuido en la calidad de la cadena productiva textil, y delincuencia en zonas aledañas.

En estos últimos 40 años, el Emporio Comercial ha crecido más de lo esperado. Este crecimiento no ha sido planificado, lo cual impacta positiva y negativamente en su desarrollo y en los alcances que tiene sobre el sector. Esto, sumado a los insuficientes esfuerzos de la sociedad civil y empresarial organizada, y de los gobernantes, ha contribuido a que hasta la fecha Gamarra no se desarrolle bajo claras estrategias que optimicen su crecimiento e impacto a nivel local, nacional e internacional. Planificación estratégica, educación, comprensión y acción son urgentes y necesarias dentro y fuera de Gamarra.

Cuenta, con un espacio de 40 cuadras, con aproximadamente 144 galerías; son alrededor de $17 \mathrm{mil}$ locales que se dedican al comercio y/o industria de la confección y los textiles, dando empleo a $60 \mathrm{mil}$ personas que unidos a los casi $10 \mathrm{mil}$ empresarios reciben diariamente $a$ un promedio de $100 \mathrm{mil}$ visitantes. Como resultado de ello se estima que este emporio textil moviliza más de 800 millones de dólares al año. Las cifras son sorprendentes no solo a nivel nacional, sino también en el espacio latinoamericano.

Se estima que en Gamarra existen cerca de 8 mil talleres con 5 máquinas por taller en promedio - donde son las mujeres la fuerza motriz - convirtiendo a este enorme centro textil peruano, en una fábrica de $40 \mathrm{mil}$ máquinas de confecciones, la más grande de América del Sur.

\section{b. Mercado Central}

La concepción del Mercado Central de Lima existe desde la creación de la ciudad en 1535 y hasta el año 1800 estuvo ubicado en la Plaza de Armas, en lo que se conoce como el Portal de Botoneros (actual Jirón 
Huallaga), frente a la Catedral de Lima. Fue en este lugar donde se ubicaron los comerciantes de productos de pan llevar y de costura, por eso el nombre de Portal de Botoneros.

En 1800 fue trasladado a la Plaza de San Francisco, donde permanecería hasta 1820, año en que se mudó a la Plaza de la Inquisición; en 1840 se instaló en las inmediaciones del Colegio Dominico de Santo Tomás y finalmente en 1851 el Presidente Ramón Castilla dispone construir el Mercado Central de Lima entre las intersecciones de los Jirones Huallaga y Ayacucho, lugar de su sede actual, obra que sería culminada durante el gobierno del General Echenique. Su construcción se realizó con una fuerte influencia europea, la que buscaba limpieza, orden y sanidad, lo que era visto como muestra de desarrollo. Ocupaba una manzana completa, de la cual se retiro al Monasterio de La Concepción. En el exterior, en los cuatro lados se construyeron locales comerciales. Los ingresos se ubicaron en la parte central de cada uno de sus lados y en sus esquinas.

En el siglo XIX se realizó la primera remodelación de su diseño original y a principios del siglo $X X$ se le hizo una ampliación. Al promediar la década de 1960 el mercado sufre un incendio y sobre este terreno se levanta el nuevo Mercado Central de Lima, tal como lo conocemos en la actualidad. En sus inmediaciones podemos encontrar al Barrio Chino situado en la calle Capón, muy solicitado por visitantes foráneos así como su variedad de frutas, verduras, cereales, etc. peruanos.

El Mercado Central cuenta con aproximadamente 47 galerías; son alrededor de 8 mil locales que se dedican al comercio, dando empleo a 40 mil personas que unidos a los casi 5 mil empresarios.

\section{c. Las Malvinas}

La zona conocida como Las Malvinas, en la avenida Argentina, Cercado de Lima, es un centro Comercial recuperado de un espacio urbano. La berma central, que había sido tomada por comerciantes informales y donde los asaltos y la delincuencia eran cosa de todos los días, ahora es un espacio completamente renovado, donde se practican diversos deportes y por el que las familias pueden caminar con tranquilidad.

Las Malvinas cuenta con un ultramoderno centro comercial de treinta mil metros cuadrados, donde se construirán 914 locales, un centro bancario, un patio de comidas, una estación policial, y que tendrá todas las facilidades necesarias para que los comerciantes y compradores realicen sus transacciones con rapidez y seguridad. "Malvinas Plaza" es el nombre de este futuro centro comercial que estará ubicado en la cuadra 6 de la avenida Argentina, una de las principales vías del centro de Lima y muy cerca de la avenida Alfonso Ugarte.

\section{ANÁLISIS, INTERPRETACIÓN}

La gestión Social Empresarial y la Gestión Ambiental (GA) afectan positivamente el desempeño de la Pymes en el emporio Gamarra, contribuyen a fortalecer la confianza, mejoran la reputación y generan valor para esas empresas. La gestión Social Empresarial y la GA tienen que ver con una visión a largo plazo; es el compromiso de la empresa por mantener un comportamiento transparente y competitivo basado en los valores, la ética y el buen gobierno corporativo, que respondan a las expectativas de los diferentes grupos de interés: accionistas, empleados, proveedores, consumidores, sociedad.

Los factores que estimulan a las organizaciones a adoptar estrategias de gestión Social Empresarial de forma pro-activa y voluntaria son intangibles pero estratégicos. Esto es lo que piensan mayoritariamente las Pymes que comienzan a tomar en serio este concepto y que han empezado a diseñar estructuras que incluyan la gestión Social Empresarial. De esta forma se han adoptado medidas que van más allá del cumplimiento legal, en temas relacionados con el buen gobierno corporativo, la mejora del entorno laboral, la acción social, el respeto al ambiente y a las prácticas comerciales.

El reto de las organizaciones es incorporar la gestión Social Empresarial y la GA dentro de la estrategia empresarial; asumir desde los altos niveles los compromisos adquiridos, evaluar los riesgos y las oportunidades que la gestión Social Empresarial y la GA representan para la organización, desarrollar indicadores de seguimiento de los objetivos planteados de gestión Social Empresarial y GA, aportar información veraz sobre las acciones realizadas, crear mecanismos de diálogo permanente con los grupos de interés que faciliten el intercambio de información sobre sus necesidades y exigencias para poder responder a ellas.

El futuro será de aquellas Pymes que se anticipen a los cambios que demanda la sociedad actual y los convierta en ventajas competitivas y en fuente de creación de valor, tanto para la organización como para todos los involucrados.

\section{PROPUESTA PARA LA SOLUCIÓN DEL PROBLEMA}

La aplicación de la gestión social en las Pymes del emporio Gamarra es muy positiva tanto para las mismas empresas, como a los grupos de interés, a continuación se brinda los principales impactos:

- Impacto en Participación Ciudadana: Con la aplicación de la gestión social empresarial se fortaleció la presencia y participación del poblador de Gamarra. Sus preocupaciones y observaciones son escuchados en pos de mejorar las prácticas empresariales que impactan directamente en su medio de influencia. 
- Impacto en Desarrollo Sostenible: Con la aplicación de la gestión social se ha podido obtener una contribución concreta, eficaz y sostenible para con el bienestar de las poblaciones asentadas en el emporio Gamarra. Además se ha podido mitigar cualquier impacto negativo, ambiental o social, que el desarrollo de su actividad empresarial puede provocar.

- Relaciones armoniosas: Con la aplicación de la gestión social se ha podido fortalecer la confianza y credibilidad entre los principales actores a través de un diálogo fluido y oportuno para asegurar la sostenibilidad de la actividad del emporio Gamarra y el desarrollo de la comunidad.

- Emisiones contaminantes: Con la aplicación de la gestión social se ha podido disminuir considerablemente las emisiones contaminantes en Gamarra.

\section{COMENTARIOS SOBRE LOS PROBLEMAS DE GESTIÓN SOCIAL EN LAS PYMES}

Si las PYMES contaran con una gestión de procesos apropiados: precio competitivo, calidad, cobertura de la fuerza de ventas, publicidad y promoción de sus productos, no generaría una falta de política comercial para su crecimiento. Esto podría deberse a la falta de apoyo del estado peruano mediante los organismos Públicos y sector financiero.

$\mathrm{Si}$ analizamos en conjunto sus problemas relacionados con la gestión social y rendimiento económico de las PYMES, resalta la falta de capacitación y la falta de una cultura de innovación y desarrollo tecnológico como los principales problemas asociados y, en principio, no son problemas la falta de liquidez, los costos de operación comercial y financiero, la falta de competitividad, ni de calidad.

Las PYMES están orientadas a satisfacer un mercado tradicional (los costos y la calidad son conocidos y controlados) donde el volumen de ventas y sus precios van a ser las variables importantes, de tal forma que por su tipo de empresa mediana y/o gran empresa es competitiva. Por otro lado, si la empresa decida orientarse hacia nuevos mercados y nuevos productos, en este caso los problemas críticos pueden tener falta de financiamiento y conocimiento de cultura tributaria y empresarial ocasionando el no crecimiento por falta de personal capacitado en sus funciones que requiera la demanda del nuevo producto o mercado generando reducción de costos que aún desconoce en el desarrollo del producto y el mercado.

Finalmente, la gestión adecuada de la comunicación permitirá un mejor relacionamiento de las Pymes con su entorno en la búsqueda de consensos. Para lo cual es necesario que las Pymes del emporio Gamarra responda y adapte mecanismos de comunicación de acuerdo a las características de los destinatarios de los mensajes, a fin de que estos puedan hacer buen uso de dicha información.

El progreso de las Pymes en el manejo de los aspectos sociales favorecerá el desarrollo de las capacidades de la población y la presencia del Estado como garante del ejercicio del derecho a la participación de la población.

Se propone que el manejo de los asuntos sociales forme parte de un sistema integrado de gestión del sector, es decir que se convierta en una estrategia transversal, en una visión de largo plazo, coherente y sistemática. Para se deben trazar los siguientes objetivos:

- Promover la unidad sectorial dinamizando e integrando la gestión social como parte esencial del desarrollo sostenible de la actividad textil.

- Realizar un manejo eficiente de casos con miras a impulsar una cultura de prevención y cooperación por vías pacíficas, sustentadas en las relaciones armoniosas de los actores del sector.

- Institucionalizar y fortalecer mecanismos y procedimientos de gestión social, manejo de casos y comunicación transparente e inclusiva.

\section{CONCLUSIONES}

- En general, las Pymes encuestadas parecen no haber asumido la visión y los valores que sustentan la Responsabilidad Social Empresarial y la Gestión Ambiental. Las organizaciones aún no han internalizando la importancia de la RSE y la GA.

- Las Pymes aún no asumen la importancia estratégica de la RSE y la GA, se aprecia debilidad en la creación de estrategias específicas de responsabilidad social.

- Las organizaciones encuestadas no desarrollan las prácticas de RSE y GA.

- El desarrollo e implantación de sistemas de indicadores que le permitan a la Pymes medir y evaluar los aspectos más intangibles ni siquiera se encuentra en formación. Ninguna de las empresas recopila información de tipo no financiero, para la elaboración de informes o memorias de RSE y GA que integren todos los aspectos que la componen.

- Los próximos desafíos para las Pymes, en el ámbito de la RSE y la GA, son: el desarrollo de estrategias de RSE y GA que consideren dentro de sus planes de acción el análisis exhaustivo de los riesgos y las oportunidades; el desarrollo de las prácticas de medición y reporte de los aspectos no financieros; la consolidación de la RSE y la GA dentro de la estructura organizativa y el involucramiento de los grupos de interés mediante diálogos permanente.

- Lograr una relación de confianza entre la sociedad y el mundo empresarial es un reto a largo plazo. La confianza se construye lentamente y para cultivarla debe haber consistencia entre lo que hacemos y lo que decimos que hacemos, así como 
de la capacidad para anteponerse y adaptarse a los cambios.

\section{AGRADECIMIENTO}

Dios por sus bendiciones y las oportunidades que me da en esta vida, por las inmensas oportunidades que me ha ofrecido para aprender, desde los libros hasta las grandes personas que me ha permitido conocer. Por la familia con que me ha bendecido, permitiéndome lograr las metas trazadas.

La UNMSM, que me abrió sus puertas y creyó en mí cuando ingresé con la esperanza de superación; a las autoridades y personal de esta noble institución, a mi maestro, verdadero catedrático que me sembró la semilla del saber y despertó en mí la necesidad de investigar para conocer la realidad del país.

Al Prof. Alvaro Ricardo Montaño Freire, quien con su ejemplo y consejos me impulsó a continuar con este artículo.

A la Dra. Ysabel Bedón Soria, por su paciencia y exigencia en cada etapa de la investigación y por haberme dedicado el tiempo necesario para asesorarme, dando como resultado la presente investigación, que espero cubra sus expectativas.

\section{REFERENCIAS}

[1] ALFONSO GOTA LOZADA, 1971, Tratado del Impuesto sobre la Renta, 3 tomos, Editorial de Derecho Financiero, Madrid.

[2] ARMANDO GEORGETI, 1967, La Evasión Tributaria, Ediciones de Palma, Buenos Aires, Argentina.

[3] ARMANDO SOLERI SIMOLER, JORGE DANOS ORDOÑEZ PALESTRA. 2006, Tema de Derecho Tributario y de Derecho Público, Libro homenaje

[4] DINO JARACH, 1983, Finanzas Públicas y Derecho Tributario, Editorial Cangallo.

[5] DURÁN ROJO, Luis. "Aplicación del Impuesto a la Renta sobre Rentas de Capital en el Perú". En: Revista Vectigalia, Año 3, № 3, Diciembre 2007. Recoge lo señalado en la Exposición de Motivos del Proyecto Legislativo.

[6] ENRIQUE G. BULIT GONE, 1986, Impuesto sobre los Ingresos Brutos, Editorial de Palma, Buenos Aires.

[7] Estudios de Derecho Internacional Tributario, 2005, Convenio de doble imposición, Editorial Instituto Colombiano de Derecho Tributario - LEGIS, Bogotá.

[8] FRANCISCO ESCRIBANO, 2009, Doctrina y Casuística de Derecho Tributario, Libro Homenaje, Grijley- AIDET.

[9] GARCÍA MULLíN, Juan Roque. Manual del Impuesto a la Renta. Centro Interamericano de Estudios Tributarios (C.I.E.T.) - DOC $\mathrm{N}^{\circ} 872$ Buenos Aires 1978. Capítulo I "Nociones Introductorias". Págs. 07 a 13.

[10] GARCíA NOVOA, César. "La influencia de la fiscalidad en las distintas formas de inversión bursátil". CEPAL. Junio 2006.

[11] HORST CLAUSE RECKPENWALD, 1970, Teoría de la Traslación de los Impuestos, Editorial de Derecho Financiero, Madrid.

[12] HORARIO A. CARCí́ BELSUNCE, Concepto de Rédito en la Doctrina y en el Derecho Tributario, Editorial Ediciones de Palma, Buenos Aires.

[13] JOHN F. DUE, 1968, Análisis económico de los Impuestos, El Ateneo, Buenos Aires.

[14] JOSÉ LUIS PÉREZ DE AYALA, Explicación de la técnica de los impuestos, zera Edición, Editoriales de Derecho Reunidas S.A.

[15] JORGE LOSSETTO, 1995, Fuentes Territorial de la Renta, Ediciones Rosgal, Montevideo.

[16] MAURICIO A. PLAZAS VEGA, 1995, El Liberalismo y la Teoría de los Tributos, Editorial Tenis S.A., Santa Fe, Bogotá.

[17] MARIO ALVA MATTEUCCI, C.PC.C. JOSÉ LUIS GARCÍA QUISPE, 2012, Aplicación Práctica del Impuesto a la Renta. 